\title{
Individual Differences, Student Satisfaction and Performance in Supplemental On-line Activities in a Postsecondary Music Course
}

\author{
Patricia Boechler, Mary Ingraham, Luis Fernando Marin, Erik deJong \\ University of Alberta, Canada
}

\begin{abstract}
This study is an extension of our previous research on the infusion of technology into a postsecondary music course to promote the skill of close listening of music. In-class hindrances in higher education classrooms, such as time, equipment, acoustics, and class size, limit student experiences of quality listening and thereby reduce their capacity for learning fundamental features important to hearing differences in musical styles.. For this study, we developed on-line, supplemental listening activities using Articulate Storyline, Adobe Connect and the virtual world Open Sim. We pretested students on music experience, computer experience and self-regulation. At the end of each course, students answered a survey on their enjoyment, tendency to recommend, their engagement and perceived increase in understanding of material, and whether or not the activities were worthwhile. In a comparison of 2014 and 2015 results, we found that students with high selfregulation levels rated these items more positively when the content included more advanced musical concepts. We also found that students who thought the on-line activities had increased their understanding of the material engaged more intensively with all the on-line activities than students who praised the convenience or aesthetic experience of the on-line activities.
\end{abstract}

\section{Introduction}

The study of music requires several learned competencies of which listening is arguably one of the most important. Listening requires intense attention to detail as well an ability to hear differences in sounds; repeated listening is therefore essential even once material is considered 'learned' and its features become familiar. Classroom situations in higher education make attentive student listening challenging not only because of the often poor equipment provided, but also the time constraints that limit the curricular space available for playing music in the context of class instruction. Despite best efforts to encourage listening outside of class, students rarely allow themselves the additional time and space needed to practice the skills learned in class. And while it is obvious that sounds are all around us, it appears that we are less and less attentive to them: it is more accurate to say that the soundscapes of our lives instead retreat to the background of much of our

day-to-day existence. The question we ask as music instructors, then, is how can we return these sounds to the foreground of attention and encourage students to listen closely to them in order to hear their differences?

In addition to the in-class hindrances noted above, music courses in higher education frequently suffer from sub-standard audio equipment and poor acoustics in classrooms, large enrolments in first year courses, diverse expectations for content (including classical, popular, folk, and world music, for example) and a range of prior experiences in performance and study of musical styles. Instructors are simply unable to expose students to enough quality listening opportunities within class time for them to be able to learn to detect the variety of musical elements considered important to their professional training [1]. To attend to these difficulties, we have developed a series of technology-based supplemental asynchronous listening activities that can be implemented on-line, outside of the classroom. These activities correspond to in-class curricular schedules while providing important opportunities for students to continue their practice of foundational music listening skills. According to Akilli [2], one of the most substantive obstacles to the optimal integration of technology into educational activities is the lack of a guiding development model that is based on learning theory and supporting research. To address this problem, we took great care in developing a theoretical framework that would guide the development of our technology-based educational activities, specifically targeted at music content [3]. This was accomplished in two steps: 1) by understanding the cognitive processes involved in the close listening of music, and 2) by employing a knowledge acquisition theory that aligned closely to those cognitive processes.

For step one, we relied on Honing's description of the cognition of music [4]. Honing's theory is based on the premise that all humans are born with the innate ability to distinguish different musical elements but through the process of learning within one's own cultural music conventions, this skill becomes implicit, that is, not available to our conscious awareness. Following Honing, then, what is needed is an approach that moves this implicit skill 
into conscious awareness, making it explicit and accessible.

For step two, we looked to Karmiloff-Smith's Representational Redescription (RR) Model [5] which explains, through a series of four stages, how knowledge moves from implicit to explicit. In the first stage, Implicit Level (I), learners hold knowledge that is not available to their conscious awareness but that allows them to correctly respond to external stimuli. Using the example of music, learners in our studies are able to detect changes in the music but do not know exactly how they are able to do so. In the second stage, Explicit Level One (E1), learners begin to understand they do indeed possess the knowledge to detect musical changes.

In Karmiloff-Smith's third stage, Explicit Level Two (E2), the emerging mental representations from E1 become more clearly articulated: learners evidence greater proficiency at detecting musical elements and begin to have the linguistic labels to share their knowledge.

The final stage, Explicit Level Three (E3), involves the highest level of redescription, in which the individual can process many forms of the evolved knowledge - in our case, knowledge of musical notation and connections between cultural artifacts related to the music to which they are

listening. Karmiloff-Smith stresses the importance of verbal interaction with others particularly in E3.

Combining these two theories to create our guiding framework, each technology was chosen to increasingly move learners' cognitive processes from implicit to explicit allowing for communication and sharing of their understanding of the musical concepts. Our first on-line activity used interactive presentations authored with Articulate Storyline to allow students to listen to pairs of music and make judgements about the musical elements they hear. This independent and self-paced listening experience is our first step in the RR Model, where the students' innate ability to hear different musical elements becomes available to their conscious awareness through repetitive listening. The second listening activity made use of Adobe Connect webconferencing software for instructor facilitated discussions. In these sessions, students use the labels for musical elements they learn from the Articulate sessions and begin expressing their understanding of the elements and their connections to the sociopolitical context from which the musical examples emerged. The last technology, the virtual world simulator Open Sim, allows for a series of peerbased discussions in an immersive environment where students, as avatars, experience cultural aspects and artefacts in multiple forms (e.g. video, audio, historical images and documents, musical scores) from the place and timeframe in which the music originated. These sessions stimulate further linguistic competency in using musical terms while students discuss their knowledge and interpretations with others.

\section{Background}

\subsection{Music Content: Basic versus Advanced}

Our study involved music history courses at two levels in our undergraduate curriculum: a first year, lecture-based course on World Music (2014; N=86) and a third-year lecture- and discussion-based course entitled "Introduction to Music in Canada" (2015; $\mathrm{N}=16$ ). The first year course incorporated what we are calling 'basic content', and the third-year course, 'advanced content'. In the Articulate listening activities for each study, musical excerpts and terminology were designed to follow in-class curriculum. Despite different course levels and requirements, basic and advanced content were presented using the same technologies. With firstyear students (2014), this included developing listening skills for recognizing and applying correct terminology to musical patterns of rhythm, meter and scale, while in the third-year course, we incorporated additional learning objects to encourage listening for

timbre (sound quality) and differences in cultural expressions (multiculturalism).

Understanding the role of music in cultural contexts is a complex, interdisciplinary activity. Advanced musical materials incorporated into our 2015 study therefore represented a more complex environment for student learning. The goal of the project with third-year students in this course was to build an understanding of the ways in which multiculturalism is expressed in music, such as the use of texts, songs, genres, or instruments from other cultures, or by incorporating musical features of these cultures such as their melodies, harmonies, rhythms or timbres. In order to identify such features, students need to be able to distinguish highly nuanced differences in musical sounds and styles, which justified introducing more conceptual topics into the Articulate activities, including advanced metrical study, nuances of timbre and listening for multiple styles of cultural expression. Recognizing meter is a fundamental skill in music education and refers to the ability to hear patterns of stressed (strong) and nonstressed (weak) beats; nonmetrical music is described as not having a regular underlying pattern of strong and weak beats. For first-year music students, hearing the musical difference between metric (regular patterns) and nonmetric (non-regular patterns) is the primary goal; in advanced listening activities such as incorporated for our third-year students in 2015, students must listen for groupings of strong and weak beats - that is, meter as a mental concept in which the music's flow constructs an overarching structure across 
longer periods of time. Further, they are required to identify whether the overall metrical organization is grouped into duple (multiples of two) or triple (multiples of three) meter, or some other combination of stressed and unstressed beats.

Timbre, or tone colour, defines the quality of a musical sound. The ability to recognize timbral difference requires students to hear sometimes nuanced sounds produced by different musical instruments. Such distinctions result from both the material from which the instrument is made as well as the manner in which the sound is produced and whether electronic enhancements are evident. While most students can (and do) hear general timbral difference (for example, the difference between a guitar and a violin), they often cannot identify the actual instrument or articulate how or why this difference is produced. Close listening to timbral difference and development of the terminology to describe sounds is thus the first step to recognition.

Identifying multiculturalism in musical expression is in many aspects even more complex in that it requires students to listen to individual and multiple features of music and to hear and comprehend the combination of diverse cultural traditions within a single work. Here we asked students to listen to and compare musical examples in which a single culture was sounded with those in which more than one cultural tradition was expressed. Without specific contextual information, students were not required to identify which cultures were heard, but rather to listen for the timbral, rhythmic, melodic, and other distinctions that could be heard as expressing one or more cultures.

Adobe Connect and Open Sim were used to present multiple cultural objects to students for discussion and comparison and to engage them in considering multiple textual, audio and visual sources across two different platforms. Here students were given materials to consider on their own, and asked to respond to questions relating to each object or to compare two or more of these objects in formulating an understanding of how musical works fit within a specific cultural context. Historical and traditional forms of expression were compared with newer ones to assist students in understanding the migration of cultural expression across time and place and to situate these within a Canadian context.

\subsection{Individual differences}

2.2.1. Prior Music Experience. Honing contends that prior music experience should not impact students' ability to engage in close listening, as this is an implicit skill. In an earlier study of the results of first-year undergraduate student listening activities, we determined that prior music experience resulted in no significant difference for student success [3]. Although it is likely that prior experience might have provided some students with a degree of comfort with terminology they were learning in the classroom at this level, the activities themselves yielded similar success for all students, regardless of any prior experience. This is good news for music instructors and students alike. However, in the current study, we compared difficulty of the content of the material which may create a difference for students with less music experiences. As an entry-level course in music, the materials included in our 2014 study were necessary to level the listening field within a very diverse classroom - to bring all of the students to a similar place of understanding of music fundamentals and their terminology such that musical styles across time and cultures could be discussed more effectively. In contrast, our 2015 students were further along in their program and, on average, had more prior music experience. Therefore, to capture any possible interactions between student differences in music experience in the context of different levels of content complexity, we again measured prior music experience.

2.2.2. Prior Computer Experience. In a review on digital literacy skills, Boechler, Dragon and Wasniewski [6] reported that levels of computer experience can be predictive of performance levels on a range of digital tasks. Because the digital literacy literature covers a wide array of definitions and measurement, and all our supplemental activities are digitally delivered, we felt it prudent to include a measure of knowledge, experiences and skills related to computer activities.

2.2.3. Self-regulation. Self-regulation refers to the capacity for self-guidance, the management of voluntary action [7], and to the self-generated thoughts, feelings, and actions that are planned and cyclically adapted to attain personal goals [8] [9] [10]. Academic self-regulation refers to students' abilities to be proactive about their own learning through their choices of motivational self-talk, strategies, learning environments and behaviours that move them toward their academic goals. Higher levels of self-regulation have been linked to higher academic achievement [11].

Self-regulated learners are reflective about their learning processes and environments, and monitor the effectiveness of their own thinking and strategies, including the types and degrees of instruction that they need [12], their active approach to learning results in activities, thoughts and feelings that help to create connections between current information and prior learning [13].

In the context of on-line learning, even stronger self-regulation skills are required to maintain motivation and engagement, and to direct effort and planning [14]. In on-line learning, although significant differences in grades of high and low self- 
regulators may be inconsistent, Artino and Stephens [15] report that low self-regulators experience less cognitive and reflective engagement as well as less satisfaction and willingness to participate in subsequent on-line experiences. Concerning music education specifically, Long, Hallam, Creech, Gaunt and Robertson reported self-regulation as a predictor of achievement in musical skill acquisition and musical performance [16] for these reasons, as in our previous research [3] we included a measure of selfregulation in the 2015 study.

This article, then, is a report of a new analysis of our 2014 articulate data and a comparison between the 2014 and 2015 articulate data, which denotes differences in students' perceptions when interacting with more advanced musical concepts in the on-line listening activities.

\section{Method}

In each study, students completed several surveys before starting the listening activities. General demographic information was collected (e.g., program, year in program, gender and age) as well as: 1) a Music Experience Survey [3], 2) a Selfregulation Questionnaire (SRQ) [17], and 3) a Computer Experience Questionnaire [6].

The Music Experience Survey asked about the music courses students may have taken and activities students engaged in outside of their coursework.

The Self-regulation Questionnaire asked students to rate statements on a 5-point Likert scale. Both positive and negative statements are included in the 63-item questionnaire such as "I usually keep track of my progress toward my goals" and "I get easily distracted from my plans".

The Computer Experience Questionnaire has three parts: 1) the Software Recognition Test (SRT), a measure of general exposure to computer applications and digital materials, 2) the Educational Activities Checklist (EAC), the number of educationrelated computer activities students have carried out (e.g., writing html code, using a formula in a spreadsheet, using a library database), and 3) the Recreational Experience Scale (RES), the number of hours per week students spent playing video games or social networking in Elementary, Junior High, High School and University.

After all on-line activities were completed, students filed out a survey of student perception of value based on a 5-point Likert scale (e.g., I enjoyed... I would recommend... I would engage in more of these activities... These activities helped my understanding... These were worthwhile activities).

\section{Results}

In a new categorical analysis on the dataset from our first study (Fall 2014), using +/- one standard deviations from their group mean as cut off points, SRQ scores were categorized into high, moderate and low self- regulation skills. For the 2014 sample $(\mathrm{N}=86$ students in a first-year undergraduate general music history course) we found that, as far as students' perceptions of the listening activities, there was a significant difference between students' perceptions across different levels of self-regulatory capacity: students more skilled in regulating themselves perceived less value in highly structured activities. Using a Likert scale where 1 equalled total disagreement and 5 total agreement, students with higher self-regulation (SRQ scores $\Rightarrow>M=213.95+$

$1 \mathrm{SD}=13.787)$ on average disagreed that they enjoyed, would recommend, or would engage in more listening activities $[\mathrm{M}=2.77, \mathrm{SD}=.928$; $\mathrm{F}(2,63)=3.877, \mathrm{p}=0.026<.05]$; that these activities had helped them improve their levels of understanding $[\mathrm{M}=2.5, \mathrm{SD}=1.41 ; \mathrm{F}(2,63)=3.613$, $\mathrm{p}=.033<.05]$; and that these were worthwhile activities in the course $[\mathrm{M}=2.25, \mathrm{SD}=1.28 ; \mathrm{F}(2,63)$ $=5.539, \mathrm{p}=0.006<.05]$.

For 2015, again we used Articulate listening activities and Open Sim Virtual Reality discussions but we also introduced an Adobe Connect Virtual Classroom discussion to present students with a variety of musical materials that would help enhance their listening and critical thinking skills. This class, a third-year undergraduate music history course, was significantly different in size $(\mathrm{n}=16)$ and composition from the 2014 dataset. On average participants were further along in years of their programs $[\mathrm{M}=4, \mathrm{SD}=$ $.81 ; \mathrm{F}(1,97)=34.421, \mathrm{p}=.000<.05]$, and more experienced in music $[\mathrm{M}=7.23, \mathrm{SD}=1.878 ; \mathrm{F}(1$, $97)=17.288, p=.000<.05]$, software recognition $[\mathrm{M}=10.77, \mathrm{SD}=3.219 ; \mathrm{F}(1,97)=15.225, \mathrm{p}=.000$ $<.05]$ and self-regulatory skills $[\mathrm{M}=223.69, \mathrm{SD}=$ 14.494; $\mathrm{F}(1,97)=6.201, \mathrm{p}=.014<.05]$. Again, based on their SRQ scores we classified our sample of students $(n=13)$ into three categories (low, middle and high) using $+/$ - one standard deviations (SD = 14.494) from their group mean $(M=223.69)$ as cut off points.

Since students who were more skilled in regulating themselves in our first sample had perceived less value in these highly structured activities, we would have expected similar or even lower results if we had used the same listening activities with the introductory concepts of rhythm and meter. After all, our second sample was significantly more experienced and with higher levels of self-regulatory ability. To put it in context, if we had used our first sample's cut off points to categorize our second sample, our data would have only loaded into middle and high levels of selfregulation. Therefore, we decided to create an additional challenge and increase the content difficulty, using more advanced concepts for the listening activities. 
We now used three activities including, again, the introductory concept of meter, to which we added two new and more advanced musical concepts: timbre and multicultural expression. We developed these new activities using the same structure of interaction, feedback and repeated opportunities for practice as the listening activities in the first study. Our results show that using these more advanced musical concepts removed differences in perceptions between students of different levels of selfregulatory capacity. On average, high SRQ students $(\mathrm{SRQ}$ scores $\Rightarrow \mathrm{M}=223.69+1 \mathrm{SD}=14.494)$ no longer disagreed but enjoyed, would recommend, or would engage in more listening activities $[\mathrm{M}=3.6$, $\mathrm{SD}=.282 ; \mathrm{F}(2,9)=0.335, \mathrm{p}=0.724>.05]$; they agreed that these activities helped them improve their levels of understanding $[\mathrm{M}=3.5, \mathrm{SD}=.707 ; \mathrm{F}(2,9)$ $=0.110, \mathrm{p}=0.897>.05]$ (see Figure 1); and that these were worthwhile activities in the course $[\mathrm{M}=$ $3.5, \mathrm{SD}=.707 ; \mathrm{F}(2,9)=0.124, \mathrm{p}=0.885>.05]$ (see Figure 2).

Even though there are no significant differences between students' perceptions across different levels of self-regulatory capacity, it is interesting to observe not only the increase in favorable perceptions of students with higher self-regulatory capacity but also a slight average decrease in students with lower SRQ capacity overall perceptions of the listening activities now that we used more advanced musical concepts (see Figures 3a, 3b).

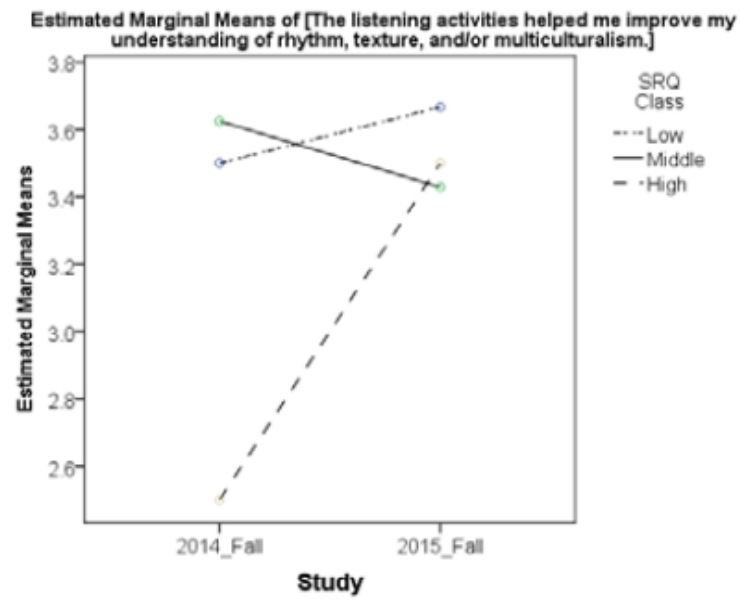

Figure 1. Students' mean rating of content understanding on-line listening activities for two different courses and levels of self-regulatory functioning

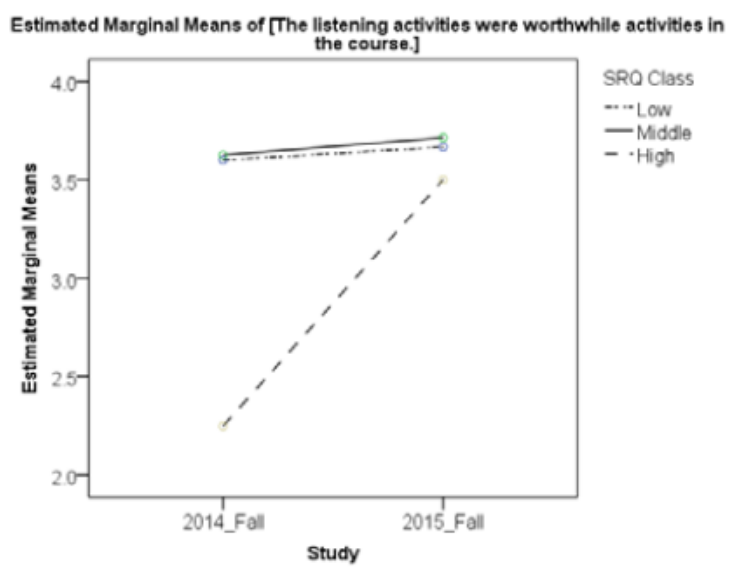

Figure 2. Students' mean rating of value to the course of on-line listening activities for two different courses and levels of self-regulatory functioning

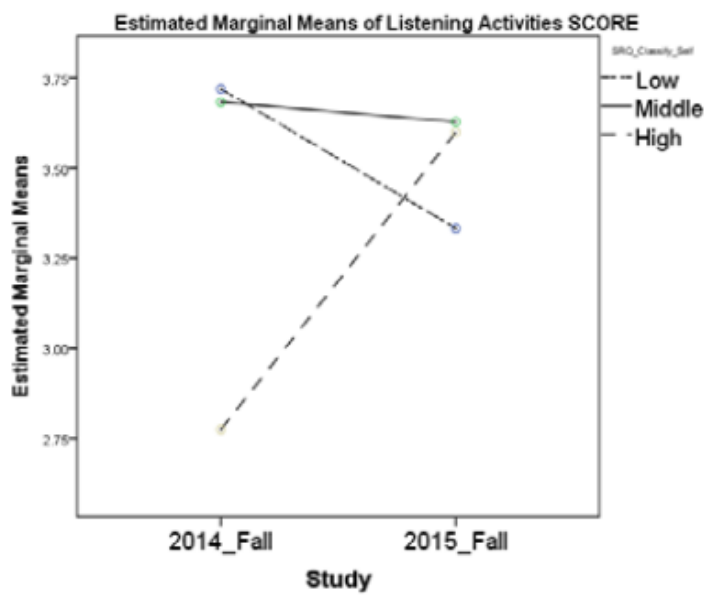

Figure 3a. Students' mean rating of on-line listening activities for two different courses and levels of selfregulatory functioning

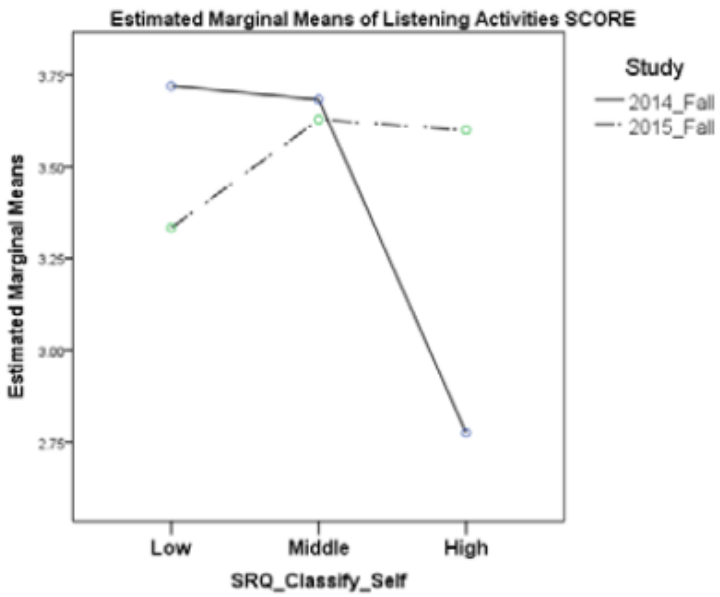

Figure 3b. Students' mean rating on survey items of on-line listening activities for different levels of selfregulatory functioning and two courses

We also asked students to complete the following sentence: "The thing I liked the most about the 
listening activities was..." In total, 58 participants (2014_Fall $\mathrm{n}=50,2015$ _Fall $\mathrm{n}=8$ ) responded to this question. We analyzed their responses and categorized them according to three themes of why they liked the activities the most: 1) convenience, 2) experience, and 3) understanding. Convenience responses $\left(n=28,2014 \_\right.$Fall $=26,2015 \_$Fall $\left.=2\right)$ included comments such as "it was easy to use," "they were short," or "took no time at all." Experience responses $\left(\mathrm{n}=19,2014 \_\right.$Fall $=16$, 2015_Fall = 3) praised aesthetic or functional features of the listening activities, e.g. "I liked the interactive experience and getting an answer right away," or "the variety in music types used," "the sound quality," and "the graphics were neat." Those students that appreciated the listening activities because they helped them improve their understanding $(\mathrm{n}=11,2014$ _Fall $=8$, 2015_Fall $=3)$ responded with comments such as "I can understand more music styles," "these were helpful to apply the things we learned in class," or "I found them to be very helpful in understanding fully certain things I was struggling with before."

In order to observe the degree of interaction we counted students' logged activities on eClass, the University's Moodle based Learning Management System (LMS). Viewing an activity, file, forum or URL, and launching an activity, or creating and submitting an assignment, are all examples of the type of events logged by eClass. We kept track of these students' interactions and created a standardized score for each student (in number of standard deviations) that helped compare their intensity of use or interaction vs the rest of the class. These standardized scores also allow us to compare the intensity of interaction between participants across our whole sample. Each one of the three courses studied here had their own unique set of activities and planned interactions, they may have required more or less intensive uses of online eClass resources, but they all had in common the same set of on-line activities: Listening Activities, Adobe Virtual Discussion (only 2015_Fall), and an OpenSim Virtual World Discussion. We kept track of all students' frequency of eClass interactions with these on-line activities and created a standardized score for each student. The listening activities represent the larger proportion (80\%) of these on-line related logged events.

To further understand the differences between different types of students in the context of music education we analyzed our eClass activities' standardized scores to address such questions as "were there any differences between high, intermediate and low self-regulators, levels of previous music experience, or levels of computer experience?" We wanted to know if high selfregulators or students with lower music experience would have had a more intense interaction with our workshop activities than their peers. We could not find any significant differences across these nine groups: low, intermediate, and high self-regulators $[\mathrm{F}(2,53)=.571=, \mathrm{p}=.568>.05]$, music experts $[\mathrm{F}(2,53)=.755=, \mathrm{p}=.475>.05]$, and computer experts $[\mathrm{F}(2,53)=2.784=, \mathrm{p}=.071>.05]$. We did find however, a significant difference in the frequency of interaction with our on-line activities between students that reported different types of positive attributes in our listening activities (see Figure 4 and 5). Students that focused on how the activities had helped them improve their understanding interacted significantly more with all of our on-line activities $(\mathrm{M}=.769, \mathrm{SD}=.225)$ than those who focused on their convenience $(\mathrm{M}=-.080$, $\mathrm{SD}=.243$, Bonff diff $\mathrm{p}=.040<.05)$, or the aesthetic and functionally pleasing aspects of the activities (M $=-.233, \mathrm{SD}=.209$, Bonff diff $\mathrm{p}=.006<.05)$, $\mathrm{F}(2,52)=5.929, \mathrm{p}=.005<.05$.

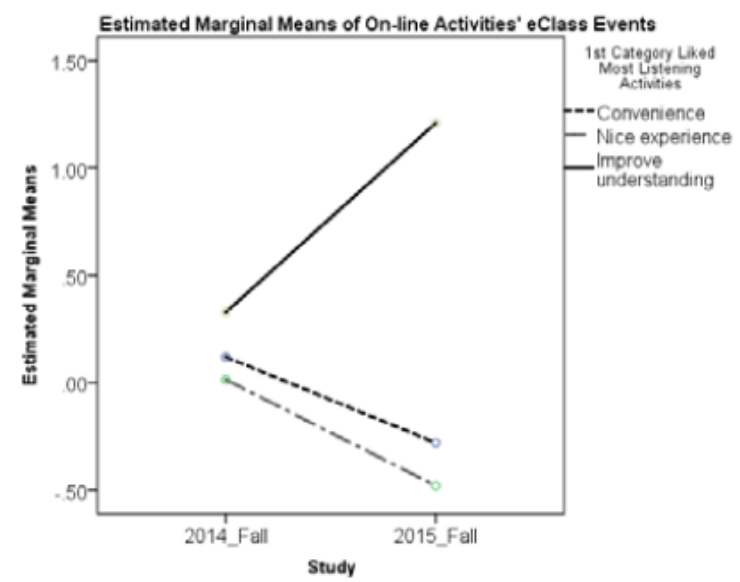

Figure 4. Students' mean intensity of interaction with the on-line activities for two studies and three different types of positive attributes found in the listening activities

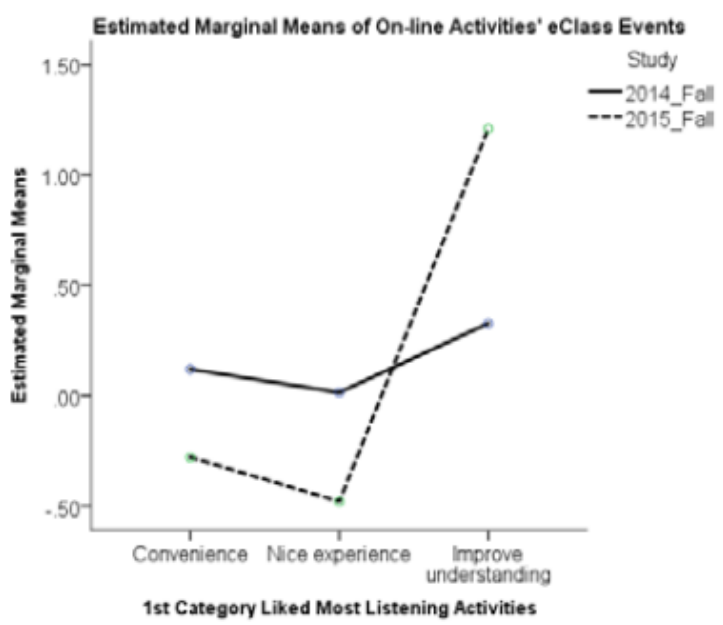

Figure 5. Students' mean intensity of interaction with the on-line activities for three different types of 
positive attributes found in the listening activities and two studies

\section{Discussion}

In this study, we found an interaction between self-regulation skills and the level of complexity of content in on-line listening activities. High selfregulators were not as satisfied with or engaged by basic musical content as were low and intermediate self-regulators but were equally satisfied when advanced content was presented.

We have referred to the overall results of the final student survey as the students' perceived value of the activities. This is comparable to what Eccles and Wigfield [18] refer to as 'task value', the degree to which students rate an activity as engaging, relevant, and beneficial. In on-line activities specifically, task value has been positively correlated to academic achievement and satisfaction, and the use of cognitive and metacognitive strategies [11].

Given that high self-regulators are strategic and reflective about what activities are more effective for reaching their academic goals, perhaps the on-line activities with basic content did not represent a high enough task value to be rated as worthwhile. To further understand the differences between high and low self-regulators in the context of music education, we will undertake subsequent analyses that address such questions as: "Did high self-regulators repeat the listening activities more than low selfregulators?" or "Did low self-regulators abandon the activity as the material became more difficult?"

Regarding students' levels of interaction with the on-line activities, we found that students who thought the on-line activities had increased their understanding of the material had engaged more intensively with all the on-line activities than students who praised the convenience or aesthetic experience of the on-line activities. This is not surprising, as understanding requires more in depth and repetitive cognitive processing than assessments of convenience or aesthetic experience.

\section{Conclusion}

The ability to listen closely is transferable to many life situations and is perhaps the most important skill required in the training of music students. It is, however, a skill that cannot be taken for granted in our modern society in which the sonic environment has become a mere backdrop to everyday life. Teaching students to listen to sounds requires considerable attention in order for them to bring the sounds they hear to the foreground, and then to be able to communicate their understanding of them in a meaningful way. Understanding the importance of music in cultural contexts adds even greater complexity to student learning, and in our undergraduate music history curriculum we lead students through four competencies at all stages of

their course work, encouraging them to develop skills such as listening for communicating their understanding of music as a unique form of cultural expression.

In our initial study of student satisfaction and performance in a blended music classroom (Fall 2014), we focused on cultivating the fundamental competency of listening in first-year undergraduate music history students; developing other competencies in reading music, critical thinking and communication were also built into this study, but were largely presented as methods for enhancing listening skills.

However, in consideration of the interdisciplinary nature of cultural studies, students interested in music must also be able to 'hear' the impact of visual materials - to interpret and critique their meaning(s) in connection with musical sounds - and to understand connections between written documentation and sounds produced within a specific social and political context. A 2007 study by cognitive psychologist Willingham suggests that critical thinking must be taught within the context of the discipline in which it is applied in order to be effective in bringing students to higher-level abilities [19]. Our experience in music education classrooms supports this position, and we have set out in these two studies to develop technology-based learning materials that would both encourage critical thinking in understanding music's place in a wider cultural context and provide opportunities for students to communicate their discoveries through peer discussion and written communication.

Pedagogically, alongside the challenges of teaching the complex role of music within specific cultural contexts, we need to be aware of the interactions between individual differences in students and the level of complexity of the content that we present. Following a comparison of these two studies, we conclude that, advanced content produced different results than basic content in relation to students' self-regulation skills.

Finally, the degree to which students interact with the on-line activities is related to their perception of the learning they experienced. Those students who engaged more intensively felt they had increased their understanding of the material. Hence, as educators we must find ways to encourage students to make use of these on-line supplemental activities.

\section{References}

[1] P. Boechler, and M. Ingraham, "The Cognition of Listening: A Cognitive Framework for the Blended Delivery of Music Curriculum." In Proceedings of the 
London International Conference on Education (LICE), Infonomics Society. London, UK, 2014, pp. 119-123.

[2] G.K. Akilli. "Games and Simulations: A New Approach to Education?" In D. Gibson, C. Aldrich \& M. Prensky (Eds.), Games and Simulations in Online Learning: Research and Development Frameworks, Information Science Publishing. Hershey, PA, 2007, pp. 120.

[3] P. Boechler, M. Ingraham, L. Fernando Marin, B. Dalen, and E. deJong, "Making the Implicit Explicit: Music Listening, Blended Delivery and the Representational Redescription Model.” In International Journal for Cross-Disciplinary Subjects in Education (IJCDSE), 6(1). Infonomics Society, London, UK, 2015, pp. 2095-2105.

[4] H. Honing, Musical Cognition: The Science of Listening. Transaction Publishers, Rutgers, NJ, 2009.

[5] A. Karmiloff-Smith, Beyond modularity: A developmental perspective on cognitive science. MIT Press, Cambridge, MA, 1992.

[6] P. Boechler, K. Dragon, and E. Wasniewski, "Digital literacy concepts and definitions: Implications for educational assessment and practice. Special Issue on Digital Literacy and Digital Competence: Facts, Problems, Needs and Trends." International Journal of Digital Literacy and Digital Competence, 5(4), 1-18. IGI Global, Hershey, PA , 2015 DOI: 10.4018/ijdldc.2014100101

[7] P. Karoly, "Mechanisms of self-regulation: A systems view." In L. W. Porter \& M. R. Rosenzweig (Eds.), Annual review of psychology, 44(1). Wiley Blackwell, Palo Alto, CA, 1993, pp. 23-52.

[8] B. J. Zimmerman, "A social cognitive view of selfregulated academic learning." Journal of educational psychology, 81(3). Francis, Ipswich, MA, 1989, p. 329.

[9] A. Bandura, "Social cognitive theory of selfregulation." Organizational behavior and human decision process, 50(2). Francis, Ipswich, MA, 1991, pp. 248-287.

[10] D. H. Schunk, and P. A. Ertmer, "Self-regulatory processes during computer skill acquisition: Goal and selfevaluative influences." Journal of educational psychology, 91(2). Francis, Ipswich, MA, 1999, p. 251.

[11] D. H. Schunk, and E. L. Usher, "Barry Zimmerman's Theory of Self-Regulated Learning." In H. Bembenutty, T. J. Cleary, \& A. Kitsantas (Eds). Applications of selfregulated learning across diverse disciplines. A tribute to Barry J. Zimmerman. Information Age Publishing, Charlotte, NC, 2013, pp. 1-28.

[12] L. Sha, C. Looi, W. Chen, P. Seow, and L. Wong, "Recognizing and measuring self-regulated learning in a mobile learning environment." In Computers in Human Behavior, 28. Science Direct, Ipswich, MA, 2012, pp. 718728.

[13] H.W. Lee, K.Y. Lim, and B. Grabowski, "Generative Learning strategies and metacognitive feedback to facilitate comprehension of complex science topics and self-regulation." In Journal of Educational Multimedia and Hypermedia, 18(1). ERIC, Ipswich, MA, 2009, pp. 5-25.

[14] N. Dabbagh, and A. Kitsantas, "Supporting selfregulation in student-centered Web-based learning environments." In International Journal on E-Learning, 3. ERIC, Ipswich, MA, 2004, pp. 40-47.

[15] A.R. Artino, and J.M. Stephens, "Beyond grades in online learning: Adaptive profiles of academic selfregulation among naval academy undergraduates." In Journal of Advanced Academics. ERIC, Ipswich, MA, 2009, pp. 568-601.

[16] M. Long, S. Hallam, A. Creech, and L. Roberston . "Do prior experience, gender, or level of study influence music students' perspectives on master classes?" Psychology of Music, 40, 6, Sage Publications, Ipswich, MA, 2011,pp. 683-699.

[17] J.M. Brown, W.R. Miller, and L. A. Lawendowski, "The Self-Regulation Questionnaire." In L. VandeCreek \& T. L. Jackson (Eds.), Innovations in clinical practice: A source book. Professional Resource Press, Sarasota, FL, 1999, pp. 281-289.

[18] J. Eccles and A. Wigfield , "In the mind of the actor: The structure of adolescents' achievement task values and expectancy-related beliefs." In Personality and Social Psychology Bulletin, 21. Sage Publications, Ipswich, MA, 1995, pp. 215-225.

[19] D.T.,Willingham, "Critical Thinking: Why is it so hard to teach?" In American Educator. American Federation of Teachers, Washington, DC, 2007, pp. 8-19. 